\title{
MKC/3-D-Histologie in der Praxis mit einem neuen Doppelskalpell - die Kokarden-Technik Ein operativer Fall mit einem Basalzellkarzinom
}

\author{
MKC/3-D-Histology in the Office Setting with a New Double Scalpel - \\ the Cockade Technique \\ A Surgical Case with a Basal Cell Carcinoma
}

Autor

Institut
G. A. Barth

Hautarztpraxis Schatz, Barth \& Degitz, München-Pasing, München
Bibliografie

Dol http://dx.doi.org/

$10.1055 / \mathrm{s}-0030-1256132$

Akt Dermatol 2011; 37:

87-90 @ Georg Thieme

Verlag KG Stuttgart · New York

ISSN 0340-2541

Korrespondenzadresse Dr. med. Georg A. Barth Hautarztpraxis Schatz, Barth \& Degitz

Pasinger Bahnhofsplatz 1

81241 München

barth@hautpraxis.de

\section{Zusammenfassung \\ $\nabla$}

Die mikroskopisch kontrollierte Chirurgie (MKC) 3-D-Histologie) ist die überlegene Therapie des Basalzellkarzinoms hinsichtlich der Rezidivhäufigkeit und Ästhetik im Vergleich zur konventio-

\section{Einleitung}

$\nabla$

Das Basalzellkarzinom ist eine der häufigsten Krebsarten des Menschen mit einer jährlichen Inzidenz von 100 Neuerkrankungen pro 100000 Einwohner in Deutschland [1]. Der bösartige Charakter beruht mehr auf dem lokal zerstörenden Wachstum als auf sehr seltenen Metastasierungen. Basalzellkarzinome entstehen typischerweise bei hellhäutigen, älteren Personen auf chronisch sonnengeschädigter Haut.

Der zunehmende Wunsch der teils gut informierten Patienten nach vollständiger Entfernung des Hauttumors einerseits und nach gewebeschonenden Verfahren mit guten funktionellen und ästhetischen Ergebnissen andererseits führt zur chirurgischen Therapie mit vollständiger mikroskopischer/histologischer Kontrolle der Schnittränder: der mikroskopisch kontrollierten Chirurgie (MKC) 3-D-Histologie).

Aufgrund des erhöhten operativen Aufwandes, der verwirrend vielfältigen Verfahren und der möglichen Komplikationen bei offenen Wunden in dem bisherigen MKC/3-D-Histologie-Verfahren hat sich diese Methode in der Breite der Praxis noch nicht durchgesetzt. Einen guten Überblick über die Vielzahl der Methoden findet man bei Moehrle et al. [2].

Hier wird ein schnelles, einfaches und komplikationsarmes operatives Vorgehen in zwei Operationen (zweizeitig) anhand eines Falles für die Praxis mit einem neuen Doppelskalpell vorgestellt - die Kokarden-Technik als Variante der MKC/3-D-Histologie. nellen Chirurgie. Die Methode hat sich aus verschiedenen Gründen in der Praxis noch nicht durchgesetzt. Wir stellen ein praxistaugliches Verfahren mit einem neuen Doppelskalpell vor die Kokarden-Technik.

\section{Operativer Fall und Methode}

$\nabla$

1. Operation (Rand-Probebiopsien, Operationszeit: 20 Minuten): Bei der 62-jährigen Patientin wurden bei einem Basalzellkarzinom an der Nase mit einem Sicherheitsabstand (SA) von $2 \mathrm{~mm}$ (Tumorrand bis zur äußeren Inzision) mit einem Doppelskalpell (Tony Knife, Fa. Tekno, Tuttlingen, Klingenabstand $1 \mathrm{~mm}$ ) die Rand-Probebiopsien (Rand-PE) genommen. Dies erfolgte jeweils in Lokalanästhesie subkutan und intrakutan mit einer Tumeszenzlösung ( 2 ml Xylonest ${ }^{\circledR} 1 \%$ mit Adrenalin $1: 200000$ und $4 \mathrm{ml} \mathrm{NaCl} \mathrm{0,9 \% ).} \mathrm{Das} \mathrm{Basalzell-}$ karzinom hatte einen Tumordurchmesser von $10 \times 10 \mathrm{~mm}$ und war bei der Erstvorstellung durch eine Shave-Probebiopsie gesichert worden.

Die erste, vertikale Doppel-Inzision mit dem Doppelskalpell (\#11 Skalpelle) wurde dabei in einem Stich-Schneid-Vorgang zur Gewinnung eines schmalen, uniformen Streifens gesetzt. Danach wurde mit einem normalen Skalpell (\#11 Skalpell) die Inzision bis in die Subkutis erweitert und der Randstreifen bei 12, 3, 6 und 9 Uhr im Verhältnis zur Körperachse durchschnitten, sodass man 4 Rand-PEs (12-3 Uhr, 3-6 Uhr, 6-9 Uhr, 9-12 Uhr) gewann. In gleicher Sitzung wurde an der Stelle des Tumors mit der am stärksten tastbaren Infiltration eine tiefe Probeexzision (tiefe PE) mit einer $2 \mathrm{~mm}$-Hautstanze (BIOPSY PUNCH von STIE$\left.\mathrm{FEL}^{\circledR}\right)$ einschließlich Subkutis genommen.

Die 4 Rand-PEs werden dann mit einem spitzen Scherchen gehoben und jeweils bei $12 \mathrm{Uhr}$ mit einer Gewebefarbe markiert. Sämtliche Probeexzisionen (4 Rand-PEs und tiefe PE) werden zur 


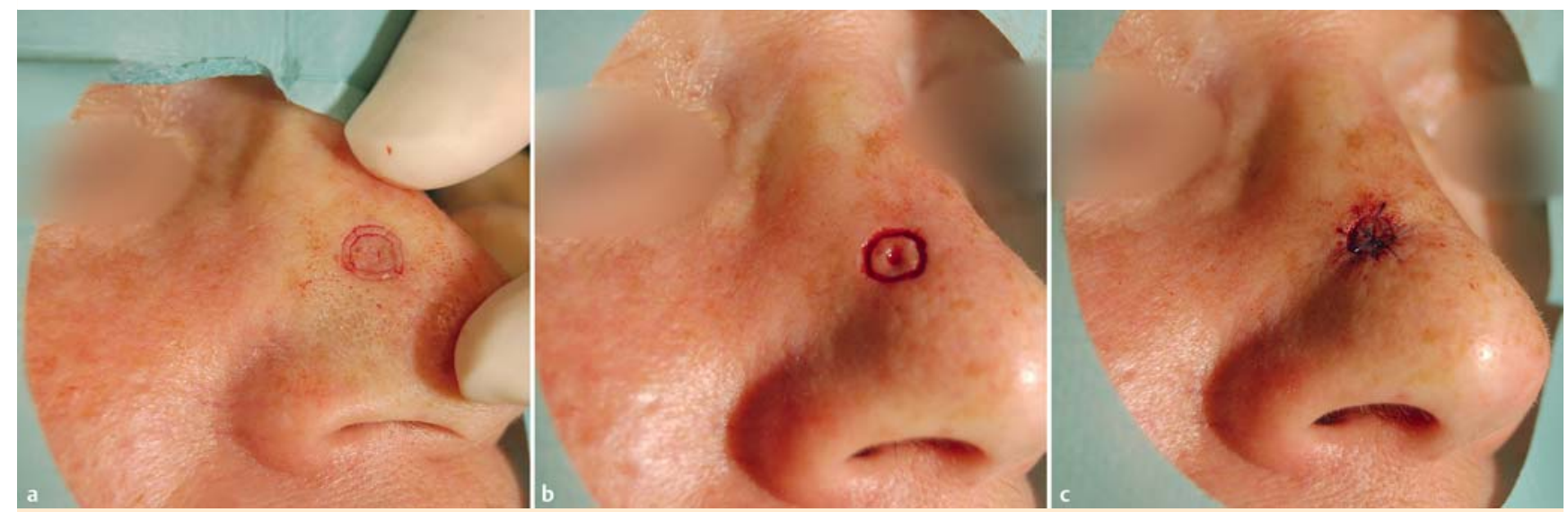

Abb. 1 (1. Operation) Links: Inzision der Rand-PEs mit dem Doppelskalpell und der tiefen PE. Mitte: Kokardenartige Defekte nach Heben der Rand-PEs und tiefen PE. Rechts: Verschluss der Defekte mittels Hautnähten.

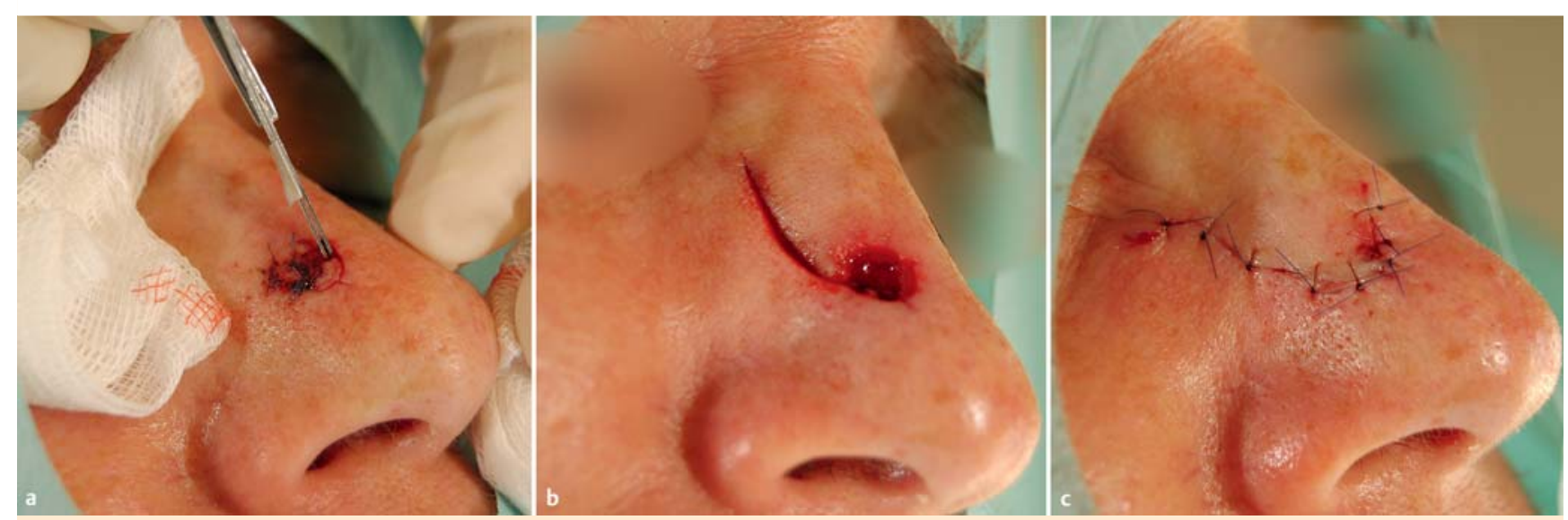

Abb. 2 (2. Operation) Links: gezielte Rand PE bei 4-5 Uhr im tumorpositiven Abschnitt mit dem Doppelskalpell. Mitte: Defekt nach Exzision des Tumors und nach Präparation des Rotationslappens. Rechts: Verschluss des Defektes mittels Rotationslappenplastik.

Routine-Histologie (Formalin-fixiert, Paraffineinbettung, HE-Färbung), zur lückenlosen mikroskopischen Randschnittkontrolle und zur mikroskopischen Untersuchung der tiefen PE verschickt. Schließlich wurden die Wunden mit fortlaufender Hautnaht, Strips und kleinem Verband verschlossen ( $\bullet$ Abb. 1).

2. Operation (10 Tage nach der 1 . Operation; gezielte Rand-PE, Exzision des Tumors und Defektdeckung; Operationszeit 45 Minuten): Entsprechend des histologischen Befundes wurde die 2. Operation geplant (Rand-PE bei $4-5 \mathrm{~h}$ war tumorpositiv; übrige Rand-PEs tumorfrei, tiefe PE zeigte ein noduläres, bis in die tiefe Lederhaut reichendes Basalzellkarzinom).

Wiederum in Tumeszenz-Lokalanästhesie erfolgte die Inzision und Hebung der Rand-PE bei 4-5 Uhr - hier also eine gezielte Nachexzision - mit einem Sicherheitsabstand von weiteren $2 \mathrm{~mm}$ (hier also $4 \mathrm{~mm}$ ) mit dem Doppelskalpell (s.o). Dann erfolgt die Exzision des gesamten Tumors entlang der äußeren frischen Narbe und einschließlich der Subkutis zur Tiefe. Die Präparate wurden bei $12 \mathrm{~h}$ markiert und in das Routine-Labor gesendet. Das Schneiden und die lückenlose mikroskopische Untersuchung der Basislamelle des Tumorexzidates erfolgte durch das Routine-Labor (histologischer Befund: alle Schnittränder tumorfrei). Nach genauer elektrokaustischer Blutstillung erfolgte die Defektdeckung mittels einer Rotationslappenlastik von kranial und mehrschichtiger Nähte (korial versenkte Nähte mit resorbierbarem Poydioxanon-Nahtmaterial sowie fortlaufende Haut- naht, die nach 7 Tagen gezogen wurde). Abschließend wurde ein Druckverband angelegt ( $\mathbf{A b b}$. 2).

\section{Diskussion}

$\nabla$

Bei der Perimeter-Technik - einer Variante der MKC/3-D-Histologie - wird erst eine mikroskopisch kontrollierte lokale Ausbreitungsdiagnostik anhand von markierten seitlichen Gewebeproben vorgenommen und die Defekte verschlossen [3]. Die Neuartigkeit der hier vorgestellten Kokarden-Methode umfasst die Entnahme der Rand-PE (Perimeter-Technik) mit einem neuen Doppelskalpell und die Entnahme einer tiefen PE zur Ausbreitungsdiagnostik des Tumors.

Die Haut im Kopf-Hals-Bereich, dort wo die meisten Basalzellkarzinome vorkommen, ist Träger einer Reihe zusätzlicher Funktionen. So ist die Beweglichkeit der Haut wichtig für die nonverbale Kommunikation (Mimik). Auch im Bereich der Augenlider, der Lippen, der Ohren und der Nasenflügel ist die Mobilität der Haut von Bedeutung für Sehen, Nahrungsaufnahme/Sprechen, Hören und Atmen.

Aufgrund der subklinischen, nur mikroskopisch sichtbaren, teils fingerförmigen (asymmetrischen) Ausbreitung von Basalzellkarzinomen über die sichtbaren seitlichen Tumorgrenzen hinaus werden seitliche Sicherheitsabstände von 3-10 mm in der kon- 
ventionellen Chirurgie empfohlen [1]. Dies führte zu großen Defekten und Verlust von gesunder Haut mit erheblichen Beeinträchtigungen der Hautbeweglichkeit durch die Bildung langer ungünstiger Narben, die aufwendige plastische Rekonstruktion notwendig machten [4-6].

Seitliche Schnittränder und Sicherheitsabstände (SA) von $4 \mathrm{~mm}$ in der MKC/3-D-Histologie und Doppelskalpelle

Mit der Einführung der lückenlosen, mikroskopischen Kontrolle der Randschnitte (MKC/3-D-Histologie) wurden kleinere Sicherheitsabstände (SA) möglich $[3,4,7,8]$.

Die MKC/3-D-Histologie führt zu signifikant kleineren Defekten und hat geringere Rezidivraten als die in der Praxis noch häufig durchgeführte konventionelle Exzision mit histologischen Querschnitten - die „Brotlaib-Methode“ [5,9-12]. Rezidive haben ein größeres Potenzial für tiefe und ausgedehnte Gewebezerstörungen als Primärtumore [13]. Kleinere Defekte ermöglichen deutlich weniger komplexe plastische Rekonstruktionen mit Vorteilen für Funktion und Ästhetik [5].

Breuninger et al. veröffentlichten bei 400 tumorpositiven Schnitträndern von primären epithelialen Tumoren (Basalzellkarzinome und Plattenepithelkarzinome), dass ein SA von $3 \mathrm{~mm}$ bei einzelnen mikroskopischen Tumorzapfen zur kompletten Entfernung in der Regel erforderlich war [4].

Ferner berichten Wolf und Zitelli bei 117 Basalzellkarzinomen bis zu einem Durchmesser von $20 \mathrm{~mm}$, dass bei einem Sicherheitsabstand von $4 \mathrm{~mm}$ vom klinisch sichtbaren Basalzellkarzinom eine vollständige Tumorentfernung in 98\% der Fälle gelingt [14]. Wir haben den aus unserer Sicht geringstmöglichen SA von $2 \mathrm{~mm}$ (1. Operation) gewählt, um möglichst viel gesunde Haut zu schonen - und dabei die Möglichkeit offen gehalten, bei Bedarf gezielt an der Stelle der tumorpositiven Rand-PE den Sicherheitsabstand auf $4 \mathrm{~mm}$ bei der Exzision und Defektdeckung (2. Operation) erweitern zu können.

Breuninger et al. wiesen zudem nach, dass bei primären Basalzellkarzinomen in einem Kollektiv von über 2000 Patienten der Anteil von tumorfreien Randschnitten nach der 2.Operation über 95\% liegt - d.h. man hat eine gute Aussicht, bei der 2. Operation mikroskopisch freie Schnittränder zu haben [15].

Deshalb haben wir eine gezielte Rand-PE (gezielte Nachexzision) - wie in unseren Fall bei 4-5 Uhr - und die Exzision des Tumors mit Defektdeckung in der 2. Operation gemeinsam durchgeführt, um dem Patienten eine zusätzliche Operation zu ersparen.

Die oben genannten Daten sind in Kliniken erhoben worden mit vielen mutmaßlichen „high risk“-Basalzellkarzinomen für Rezidive (Rezidiv-Basalzellkarzinome, Tumore > 20 mm; sklerodermiformes Basalzellkarzinom; Lokalisation Nase, Anamnese länger als 1 Jahr, breiter tumorpositiver Randschnitt) [4,6]. Im ambulanten Patientengut sind diese Formen seltener und Rand-PEs mit einem SA von $4 \mathrm{~mm}$ mutmaßlich seltener tumorpositiv.

Mit dem Doppelskalpell kann man einfach und schnell uniforme Hautstreifen gewinnen - so auch Rand-PEs zur lateralen mikroskopischen Kontrolle bei Hauttumoren.

Doppelskalpelle sind in der Chirurgie nicht neu. Coiffman veröffentlichte 1977 den Einsatz eines Doppelskalpells in der Haartransplantation, später kamen weitere Beschreibungen mit unterschiedlichen Indikationen wie Narbenkorrektur und Exzision von Hauttumoren hinzu [16-21].

Die Alternative, nämlich mit der freien Hand geschnittene Randproben, ist häufig ungenau und zeitaufwendig, speziell bei schmalen Randproben. Gerade die zweite, parallel mit der Hand geschnittene Inzision ist durch die fehlende Hautspannung bei einem Abstand von $1 \mathrm{~mm}$ kaum präzise zu schneiden.

Die bisherigen Doppelskalpelle sind in der Handhabung durch ungewohnte Griffe schwer führbar, außerdem durch aufwendige Konstruktionen und Einzelanfertigungen nicht verfügbar und fangen erst bei $1,5 \mathrm{~mm}$ Schnittbreite an.

Der entscheidende Vorteil des neuen, von uns entwickelten Doppelskalpells ist - unseres Erachtens - neben der einfachen Handhabung (gewohnter Griff; leichtes Einspannen der Klingen) die möglichst große Gewebeschonung durch die stufenlos einstellbaren Abstände zwischen 0-2 mm für die Rand-PE.

\section{Basaler Schnittrand in der MKC/3-D-Histologie}

Zusätzlich zu den Rand-PEs mit dem Doppelskalpell führen wir eine tiefe PE an der Stelle mit der stärksten tastbaren Infiltration durch, um die Tiefenausdehnung auszuloten. Im Falle einer seltenen tiefen, mikroskopisch nachweisbaren Infiltration des Tumors weit in die Subkutis sollte ein alternatives operatives Vorgehen bei der 2. Operation erwogen werden (z. B. stationäre Therapie). Bei der 2. Operation besteht eine hohe Wahrscheinlichkeit der kompletten Tumorentfernung zur Tiefe, da bei Primärtumoren in ca. $95 \%$ bei Mitentfernung der Subkutis eine vollständigen Entfernung zur Tiefe erfolgt $[4,15]$.

Ein möglicher Nachteil unserer Methode besteht in der lückenlosen mikroskopischen Kontrolle des basalen Schnittrandes erst nach der Exzision und Defektdeckung.

Trotz eines mutmaßlich geringen Risikos für eine ausgedehnte Tiefeninfiltration im ambulanten Patientengut dokumentieren wir anhand von Fotos die bedeutsamen Lokalisationen wie tumorpositver Schnittrand und basaler Schnittrand auch nach der Defektdeckung für eine unwahrscheinliche Nachoperation.

\section{Vorteile der Kokarden-Methode}

Das meist zweizeitige (1. und 2.Operation) Vorgehen im Vergleich zur einzeitigen konventionellen Chirurgie ohne MKC/3-DHistologie ist aufwendiger - wird jedoch durch die kontrollierte vollständige Exzision mit geringeren Rezidivraten und kleineren Defekten mehr als ausgeglichen.

Offene Wunden für Tage, wie sie häufig nach Exzision des Tumors bei der 1. Operation nach MKC/3-D-Histologie im bisherigen Verfahren vorliegen, haben entscheidende Nachteile: Sie sind stark exudativ und machen häufige und aufwendige Verbandwechsel notwendig; sie bilden Granulationsgewebe und Fibrinbeläge.

Da bei der Kokarden-Methode die Wunden verschlossen werden, liegen folgende Vorteile auf der Hand:

- Die Operationstermine können nach den Wünschen des Patienten und nach den Praxisgegebenheiten flexibel vergeben werden, weil keine offene Wunde zeitnah versorgt werden muss.

- Kleine, unauffällige Verbände bei der Kokarden-Technik führen zu einer erhöhten Patientenzufriedenheit.

- Kein störendes Granulationsgewebe und Fibrinbeläge in der offenen Wunde, die bei der Defektdeckung erst operativ entfernt werden müssen.

- Besonders wichtig erscheint uns folgender Aspekt der Kokarden-Methode. Offene Wunden sind anfälliger für Komplikationen wie Infektionen und Nachblutungen - besonders unter Einnahme von Antikoagulantien. In der Praxis sind wir zunehmend mit älteren Patienten mit Hauttumoren konfrontiert, die Antikoagulantien (ASS, Clopidogrel, Marcumar) einnehmen müssen. 
Die Kokarden-Technik erlaubt in geübter Hand die kontrollierte Entfernung des Hauttumors und die Defektdeckung unter Medikamenten der Blutverdünnung durch den zusätzlich blutstillenden Effekt mehrschichtiger Nähte.

Auch an Lokalisationen wie Nasenflügel, Lippen und Lider, an denen der zusätzliche blutstillende Druckverband bei offenen Wunden schwer möglich ist, ist die Kokarden-Technik eine gute Möglichkeit, die Hauttumore zu entfernen - von anderer Stelle wurde in diesen Fällen die in der Praxis in der Regel nicht vorhandene Kryostat-Methode empfohlen [22].

Prinzipiell ist die Kokarden-Technik auch bei anderen Hauttumoren (z.B. Plattenepithelkarzinom, Spinaliom) möglich.

Man kann nur mutmaßen, warum sich die MKC/3-D-Histologie mit den Vorteilen für Funktion und Ästhetik bei Basalzellkarzinom im Kopf-Bereich in der Breite der Praxis nicht durchgesetzt hat. Möglicherweise - wie auch in anderen Ländern - gibt es versicherungstechnische und operationsbedingte Gründe [16,23].

Die 1. Operation ist beispielsweise eine ambulante/ggf. teilstationäre Maßnahme, die leider bei gesetzlich versicherten Patienten schlecht abzurechnen ist. Möglicherweise lässt sich diese - nach gründlicher Aufklärung des Patienten über die Vorteile - „IGeLn“. In der Privatmedizin kann man die Kokarden-Methode wirtschaftlich darstellen.

Unseres Erachtens aber liegt es auch an den verwirrend vielfältigen Methoden, teils mit hohem zeitlichen und apparativen Aufwand, die häufig im täglichen ambulanten Praxisbetrieb schwer integrierbar sind. Auch die Furcht des Operateurs vor Infektionen und Nachblutungen bei offenen Wunden unter ambulanten Bedingungen könnte ein Grund sein.

Mit der hier beschriebenen Kokarden-Methode haben wir anhand eines Falls ein praxistaugliches, einfaches, schnelles und komplikationsarmes Verfahren für die Exzision von Basalzellkarzinomen in zwei Operationen - auch bei einem Patienten unter Antikoagulation - mit den Vorteilen der MKC/3-D-Histologie vorgestellt.

\section{Danksagung}

Für die freundliche Durchsicht des Manuskriptes möchte ich mich bei meinen Kollegen Dr. Harald Schatz, Prof. Dr. Klaus Degitz und Dr. Michael Gummer bedanken.

Abstract

\section{MKC/3-D-Histology in the Office Setting with a New Double Scalpel - the Cockade Technique \\ $\nabla$}

The microsopically controlled surgery (MKC/3-D-histology/slow Mohs surgery) is the superior therapy regarding recurrence und aesthetic outcome in comparison to conventional surgery.

Due to different reasons this method is not performed broadly.

We are presenting a procedure for the office setting with a new double scalpel - the cockade technique.

\section{Literatur}

1 Reifenberger J, Ruzicka T. Basal Cell Carcinoma. In: Burgdorf WHC, Plewig G, Wolff HH, Landthaler M, Hrsg. Braun-Falco's Dermatology. (3rd edition). Heidelberg: Springer Medizin; 2009

2 Moehrle M, Breuninger H, Röcken M. A confusing world: What to call histology of three-dimensional tumour margins? J Eur Acad Dermatol Vernerol 2007; 21: $591-595$

3 Mahoney MH, Joseph M, Temple CL. The perimeter technique for lentigo maligna: an alternative to Mohs micrographic surgery. J Surg Oncol 2005; $1: 120-125$

4 Breuninger H, Doh S, Dietz K. Sicherheitsabstände bei der Exzision von malignen epithelialen Hauttumoren. In: Rompel R, Petres J, Hrsg. Operative onkologische Dermatologie. Berlin/Heidelberg/New York: Springer; 1999: 145-149

5 Muller FM, Dawe RS, Moseley H, Fleming CJ. Randomized comparison of Mohs micrographic surgery and surgical excision for small nodular basal cell carcinoma. Tissue-sparing outcome. Dermatol Surg 2009; 35: $1349-1354$

6 Woerle B, Heckmann M, Konz B. Micrographic surgery of basal cell carcinomas of the head. Recent Results Cancer 2002; 160: 219-224

7 Johnson TM, Headington JT, Baker SR, Lowe L. Usefulness of staged excision for lentigo maligna and lentigo maligna melanoma: The square procedure. J Am Acad Dermatol 1997; 37: 758 - 764

8 Petres J, Rompel R. Operative Dermatologie. 2. Aufl. Heidelberg: Springer; 2007: 125-127

9 Eberle FC, Schippert W, Trilling B et al. Cosmetic results of histographically excision of non-melanoma skin cancer in the head and neck region. JDDG 2005; 3: 109-112

10 Rowe DE, Carroll RJ, Day CLJr. Long-term recurrence rates in previously untreated (primary) basal cell carcinoma: implications for patient follow-up. J Dermatol Surg Oncol 1989; 15: 315 - 328

11 Rowe DE, Carroll RJ, Day CL Jr. Mohs surgery is the treatment of choice for recurrent (previously treated) basal cell carcinoma. J Dermatol Surg Oncol 1989; 15: 424-431

12 Thissen MRTM, Neumann MHA, Schouten LJ. A systematic review of treatment modalities for primary basal cell carcinomas. Arch Dermatol 1999; 135: $1177-1183$

13 van Baardwijk AA, Verhaegh ME, Krekels GA et al. Mohs method of micrographic surgery as treatment for recurrent basal call carcinoma. Ned Tijdschr Genoeskd 1997; 141: 524-529

14 Wolf DJ, Zitelli JA. Surgical margins for basal cell carcinoma. Arch Dermatol 1987; 123: $340-344$

15 Breuninger H, Schippert W, Black B, Rassner G. Untersuchungen zum Sicherheitsabstand und zur Exzisionstiefe in der operativen Behandlung bei 2016 Tumoren. Hautarzt 1989; 40: 693-700

16 Aoyagi S, Hata H, Homma E, Shimizu H. Controlling the histological margins for nonmelanoma skin cancer conveniently using a doublebladed scalpel. J Surg Oncol 2010; 101 (2): 175-179

17 Bowen ML, Charnock FM. The Bowen double-bladed scalpel for the reconstruction of scars. Obest Gynecol 1994; 83: 476-477

18 Cernea CR, Velasco O, Gomes MQT et al. Double-bladed scalpel. A new option for harvesting margins in head and neck cancers. Journal for Oto-Rhino-Laryngology (ORL), Head and Neck Surgery 2006; 68: 83 87

19 Chen TM, Wanitphakdeedecha R, Nguyen TH. Multibladed knife for staged surgical margin control in nonmelanoma skin cancer. Plast Reconstr Surg 2008; 121: $1870-1871$

20 Coiffman F. Use of square scalp grafts for male pattern baldness. Plast Reconstr Surg 1977; 60: 228-232

21 Schultz BC, Roenigk HH Jr. The double scalpel and double punch excision of skin tumors. J Am Acad Dermatol 1982; 7: 495-499

22 Stein A, Büchner L, Hackert I, Sebastian G. Die „Doppelte Flunder“ zur Aufarbeitung größerer Tumorexzisate in Kryostattechnik. In: Rompel R, Petres J, Hrsg. Operative onkologische Dermatologie. Berlin/Heidelberg/New York: Springer; 1999: 139-144

23 Takenouchi T, Nomoto S, Ito M. Factors influencing the linear depth of invasion of primary basal cell carcinoma. Dermtol Surg 2001; 27: $393-396$ 